\title{
Gene expression profiles of ductal versus acinar adenocarcinoma of the prostate
}

\author{
Souzan Sanati ${ }^{1}$, Mark A Watson ${ }^{2}$, Andrea L Salavaggione ${ }^{2}$ and Peter A Humphrey ${ }^{1}$ \\ ${ }^{1}$ Department of Pathology and Immunology, Division of Anatomic and Molecular Pathology, Washington \\ University School of Medicine, St Louis, MO, USA and ${ }^{2}$ Department of Pathology and Immunology, Division \\ of Laboratory and Genomic Medicine, Washington University School of Medicine, St Louis, MO, USA
}

\begin{abstract}
Ductal adenocarcinoma is an uncommon variant of prostatic adenocarcinoma with a generally more aggressive clinical course than usual acinar adenocarcinoma. However, the molecular distinction between ductal and acinar adenocarcinomas is not well characterized. The aim of this investigation was to evaluate the relatedness of ductal versus acinar prostatic adenocarcinoma by comparative gene expression profiling. Archived, deidentified, snap frozen tumor tissue from 5 ductal adenocarcinomas, 3 mixed ductal-acinar adenocarcinomas, and 11 acinar adenocarcinomas cases were analyzed. All cases of acinar and ductal adenocarcinomas were matched by Gleason grade. RNA from whole tissue sections of the 5 ductal and 11 acinar adenocarcinomas cases were subjected to gene expression profiling on Affymetrix U133Plus2 microarrays. Independently, lasercapture microdissection was also performed on the three mixed ductal-acinar cases and five pure acinar cases to isolate homogeneous populations of ductal and acinar carcinoma cells from the same tumor. Seven of these laser-capture microdissected samples (three ductal and four acinar cell populations) were similarly analyzed on U133Plus2 arrays. Analysis of data from whole sections of ductal and acinar carcinomas identified only 25 gene transcripts whose expression was significantly and at least two-fold different between ductal and acinar adenocarcinomas. A similar analysis of microdissected cell populations identified 10 transcripts, including the prolactin receptor, with more significant differences in expression of 5- to 27-fold between ductal and acinar adenocarcinomas cells. Overexpression of prolactin receptor protein in ductal versus acinar adenocarcinoma was confirmed by immunohistochemistry in an independent set of tumors. We conclude that ductal and acinar adenocarcinomas of the prostate are strikingly similar at the level of gene expression. However, several of the genes identified in this study, including the prolactin receptor, represent targets for further investigations on the molecular basis for histomorphological and clinical behavioral differences between acinar and ductal adenocarcinomas.
\end{abstract}

Modern Pathology (2009) 22, 1273-1279; doi:10.1038/modpathol.2009.103; published online 24 July 2009

Keywords: prostate; neoplasms; ductal; adenocarcinoma; gene; prolactin

Ductal adenocarcinoma is the most common histologic variant of prostatic carcinoma. ${ }^{1-10}$ Most studies have shown a more aggressive clinical course for ductal adenocarcinoma compared with usual acinar adenocarcinoma. ${ }^{6-14}$ There has been debate as to whether ductal adenocarcinoma is an entity that is distinct from acinar adenocarcinoma. ${ }^{1,5}$ Ductal adenocarcinomas are often admixed with acinar adenocarcinoma and ductal and acinar adenocarcinomas can share certain histopathological attributes

Correspondence: Dr PA Humphrey, MD, PhD, Department of Pathology and Immunology, Division of Anatomic and Molecular Pathology, Washington University Medical Center, 660 South Euclid Avenue, Campus Box 8118, St. Louis, MO 63110, USA.

E-mail: humphrey@path.wustl.edu

Received 14 April 2009; revised 23 June 2009; accepted 26 June 2009; published online 24 July 2009 such as cribriform growth and origin in the peripheral zone. Yet, there are also differences in that ductal adenocarcinoma commonly arises in large periurethral ducts, displays a papillary growth configuration, and characteristically is comprised of tall pseudostratified columnar epithelial cells. Despite these differences, it has been proposed that ductal adenocarcinoma results from spread of typical acinar adenocarcinoma into large accommodating periurethral ducts and stroma, ${ }^{5}$ although this is a minority view. The World Health Organization tumor classification book $^{3}$ and the AFIP fascicle ${ }^{4}$ currently recognize ductal adenocarcinoma as a variant and subtype of prostatic adenocarcinoma, respectively.

The molecular relatedness of ductal and acinar adenocarcinomas of the prostate has been addressed 
in only a few studies. Limited scale data do exist on comparative protein expression in ductal versus acinar adenocarcinoma, ${ }^{7,12,14-25}$ but comparative global gene expression profiling has not been performed. The aim of this study was to examine the gene expression profiles of ductal versus acinar adenocarcinoma to assess the molecular relatedness of ductal and acinar adenocarcinomas and to identify potential molecular differences that may explain the different histopathological features and disparate clinical behavior of ductal adenocarcinoma of the prostate.

\section{Materials and methods}

\section{Human Tissues}

After IRB/human studies approval, archived, deidentified, snap frozen tumor tissue from 5 cases of ductal adenocarcinoma and 11 cases of acinar adenocarcinoma matched for Gleason grade (mean Gleason score of 8 for ductal cases and 8.2 for acinar cases) were obtained from the Siteman Cancer Center Tissue Procurement Core Facility for RNA isolation and gene expression microarray analysis. Frozen sections of banked tissue were reviewed for tumor cellularity, Gleason grade, and histological type. Whole tissue sections used for RNA extraction had a mean of $65 \%$ (range $25-100 \%$ ) of the tissue cellularity involved by carcinoma. Formalin-fixed, paraffin-embedded blocks of ductal and acinar adenocarcinomas used for immunohistochemistry were identified by a computerized search of the surgical pathology files at Barnes-Jewish Hospital.

\section{RNA Isolation from Frozen Tissues and Microarray Analysis}

Serial $50 \mu \mathrm{m}$ sections were homogenized in TRIzol reagent (Invitrogen, Carlsbad, CA, USA) to extract total RNA from whole tissue ductal $(n=5)$ and acinar tumor samples $(n=11)$. Laser-capture microdissection from frozen sections of three additional mixed acinar and ductal adenocarcinomas cases and four Gleason grade matched acinar adenocarcinomas was performed using the Arcturus Pixcell II instrument. We did not separately identify and microdissect intraductal components of ductal adenocarcinomas. RNA isolation from microdissected ductal and acinar cell populations was performed using the RNA Micro Isolation kit (Stratagene, La Jolla, CA, USA) as described earlier. ${ }^{26}$ All RNA was quantified by Nanodrop spectrophotometry (Thermo Fisher Scientific) and qualitatively assessed using an Agilent Bioanalyzer (Agilent, Santa Clara, CA, USA).

Five micrograms of RNA from each whole tumor section was converted to biotinylated, antisense cRNA target by the Siteman Cancer Center Multiplexed Gene Analysis Core, using Affymetrix GeneChip reagents and the manufacturer's standard protocol (Affymetrix, Santa Clara, CA, USA). Fifty nanograms of RNA from microdissected cell populations was independently converted to biotinylated, antisense cRNA target, using the Affymetrix two-cycle labeling method. All biotinylated targets were fragmented and $15 \mu \mathrm{g}$ of each was hybridized to HG-U133Plus 2.0 GeneChip microarrays following the manufacturer's protocol. Scanned array images were reviewed and converted to signal data using the Affymetrix MAS 5.0 algorithm, scaling each array set (total tissue RNA and microdissected RNA) independently to a target intensity of 1500 . Scaled data for each array were exported to the Siteman Cancer Center Bioinformatics server (http:// bioinformatics.wustl.edu), merged with the updated gene annotation data for each probe set on the array, and downloaded for further data visualization and analysis. The completely annotated, minimum information about a microarray experiment-compliant dataset can be found at the above noted URL and also at Gene Expression Omnibus (http:// www.ncbi.nlm.nih.gov/geo/).

\section{Data Analysis}

Basic microarray data visualization, data filtering, ANOVA analysis, and hierarchical clustering were done using Spotfire DecisionSite for Functional Genomics (Somerville, MA, USA). The $P$-values obtained from ANOVA were corrected for multiple testing based on the method of Benjamini-Hochberg. ${ }^{27}$ Data sets from whole tissue sections and microdissected cell populations were treated independently, as they involved two different methods of target preparation.

Microarray probe sets that were scored 'absent' across all arrays and Affymetrix control probe sets were removed from further analysis. For unsupervised hierarchical clustering using Ward's method, signal values for each probe set across arrays were normalized by the Z-score method and relative expression was used to generate clusters. For comparative analysis, the significance analysis of microarrays algorithm ${ }^{28}$ was used to identify differentially expressed transcripts between two, unpaired classes (acinar and ductal) with the minimum possible false discover rate. Secondarily, a simple two-class ANOVA analysis was performed to calculate $P$-values, which were subsequently corrected for multiple comparisons. Lists of significantly different transcripts were further filtered by the criteria that expression values (signal levels) were at least two-fold different, in anticipation of the need to confirm results using less quantitative immunohistochemistry.

\section{Immunohistochemistry}

An independent set of archival formalin-fixed, paraffin-embedded tissue from 20 cases of mixed ductal-acinar adenocarcinomas of prostate was retrieved for immunohistochemical staining using 
a mouse monoclonal antibody reactive with the prolactin receptor (prediluted clone SPM2123; Spring Bioscience, Fremont, CA, USA). The tissues were from radical prostatectomy cases from 1995 to 2003. The primary antibody incubation was for $0.5 \mathrm{~h}$ at room temperature after antigen retrieval followed by Vectastain ABC development (Vector Laboratories, Burlingame, CA, USA). The positive tissue control was breast carcinoma and a positive staining signal was cytoplasmic. Visual inspection grading was performed by assessment of intensity of staining as negative (0), weak $(1+)$, moderate $(2+)$, or strong $(3+)$. The percentage of stained tumor cells was graded as $\leqslant 10 \%(1+)$, 11$50 \%(2+), 51-79 \%(3+)$, and $\geqslant 80 \%(4+)$. The immunoreactive score was determined as the product of the intensity and percentage of staining. The maximum assigned immunoreactive score was 12 .

\section{Results}

Among the 16 whole tumor tissue sections analyzed, unsupervised hierarchical cluster analysis based on the entire set of expressed probes ( $\sim 38000$ probes $)$ showed no consistent, global difference in the gene expression profile between acinar adenocarcarcinoma $(n=11)$ and ductal adenocarcinoma $(n=5)$. To determine whether such a distinction could be better observed at a cellular level, a similar analysis of a smaller set of seven microdissected cell populations was performed. However, expression of the $\sim 37000$ probes in this sample set also did not distinguish acinar cells $(n=4)$ from ductal cells $(n=3)$. These data suggest no global differences in gene expression between these two cell types.

To identify specific differences in gene expression between acinar and ductal tumors, we performed both simple ANOVA analysis as well as a more stringent significance analysis of microarrays analysis. We identified a total of 41 characterized transcripts with significant differential expression $(P<0.05$, correct for multiple comparisons), but only 14 of these transcripts showed a differential expression level of greater than two-fold. Furthermore, applying significance analysis of microarrays analysis to the whole data at a minimum false discovery rate of $20 \%$ confirmed only nine of these transcripts (Table 1).

Table 1 Differential gene expression between ductal and acinar adenocarcinoma of the prostate

\begin{tabular}{|c|c|c|c|}
\hline Gene symbol & Gene name & P-value & $\begin{array}{c}\text { Fold difference } \\
\text { (acinar versus ductal) }\end{array}$ \\
\hline RRBP1 & ribosome binding protein 1 homolog $180 \mathrm{kDa}(\mathrm{dog})$ & $2.4 \mathrm{E}-06$ & 0.5 \\
\hline BOLL & bol, boule-like (Drosophila) & 0.0001 & 0.5 \\
\hline ZNF354C & zinc finger protein $354 C$ & 0.0002 & 1.2 \\
\hline KIAA0703 & KIAA0703 gene product & 0.0003 & 0.4 \\
\hline CCNI & cyclin $I$ & 0.0006 & 1.5 \\
\hline LDLR & low density lipoprotein receptor (familial hypercholesterolemia) & 0.0008 & 0.3 \\
\hline CXorf53 & chromosome $X$ open reading frame 53 & 0.001 & 0.3 \\
\hline PTPRF & protein tyrosine phosphatase, receptor type, $F$ & 0.002 & 0.6 \\
\hline LRP10 & low density lipoprotein receptor-related protein 10 & 0.002 & 0.5 \\
\hline PPP1R7 & protein phosphatase 1 , regulatory subunit 7 & 0.002 & 0.2 \\
\hline NUMB & numb homolog (Drosophila) & 0.003 & 1.8 \\
\hline TCN1 & transcobalamin I (vitamin B12 binding protein, $R$ binder family) & 0.003 & 0.1 \\
\hline C14orf32 & chromosome 14 open reading frame 32 & 0.004 & 0.7 \\
\hline PRDM2 & $P R$ domain containing 2 , with $Z N F$ domain & 0.005 & 0.7 \\
\hline KIAA1217 & KIAA1217 & 0.005 & 0.4 \\
\hline C14orf32 & chromosome 14 open reading frame 32 & 0.006 & 0.7 \\
\hline SNRK & $S N F-1$ related kinase & 0.007 & 0.4 \\
\hline LDLR & low density lipoprotein receptor (familial hypercholesterolemia) & 0.007 & 0.5 \\
\hline NCOA3 & nuclear receptor coactivator 3 & 0.008 & 0.6 \\
\hline PRKCBP1 & protein kinase $C$ binding protein 1 & 0.008 & 0.3 \\
\hline C14orf2 & chromosome 14 open reading frame 2 & 0.009 & 1.7 \\
\hline C3orf15 & chromosome 3 open reading frame 15 & 0.01 & 0.4 \\
\hline RNF38 & ring finger protein 38 & 0.01 & 0.6 \\
\hline SKIIP & SKI interacting protein & 0.01 & 1.4 \\
\hline MAML2 & mastermind-like 2 (Drosophila) & 0.01 & 0.5 \\
\hline PPP2R5C & phosphatase 2 , regulatory subunit $B$ (B56), gamma isoform & 0.02 & 2.6 \\
\hline GDA & guanine deaminase & 0.02 & 0.3 \\
\hline PLXNB1 & plexin $B 1$ & 0.02 & 0.4 \\
\hline REC14 & recombination protein $\mathrm{REC14}$ & 0.02 & 1.6 \\
\hline STARD5 & START domain containing 5 & 0.02 & 0.6 \\
\hline PTPN3 & protein tyrosine phosphatase, non-receptor type 3 & 0.03 & 0.5 \\
\hline AKAP13 & A kinase (PRKA) anchor protein 13 & 0.03 & 0.6 \\
\hline MUC1 & mucin 1 , transmembrane & 0.03 & 0.3 \\
\hline MID1 & midline 1 (Opitz/BBB syndrome) & 0.03 & 0.5 \\
\hline PKHD1L1 & polycystic kidney and hepatic disease 1 -like 1 & 0.03 & 3.0 \\
\hline FLJ10300 & hypothetical protein FLJ10300 & 0.03 & 0.5 \\
\hline CNIH & cornichon homolog (Drosophila) & 0.04 & 1.3 \\
\hline ANXA11 & annexin A11 & 0.04 & 0.5 \\
\hline C14orf2 & chromosome 14 open reading frame 2 & 0.05 & 1.7 \\
\hline CHST10 & carbohydrate sulfotransferase 10 & 0.05 & 0.7 \\
\hline MID1 & midline 1 (Opitz/BBB syndrome) & 0.05 & 0.5 \\
\hline
\end{tabular}

Genes in bold demonstrate significant difference in expression by both ANOVA and significance analysis of microarrays analyses. 
Table 2 Differential gene expression between microdissected ductal and acinar cell populations in adenocarcinoma of the prostate

\begin{tabular}{|c|c|c|c|}
\hline Gene symbol & Gene name & P-value & $\begin{array}{c}\text { Fold difference } \\
\text { (ductal versus acinar) }\end{array}$ \\
\hline LCN2 & lipocalin 2 (oncogene 24p3) & $2.7 \mathrm{E}-06$ & 17.4 \\
\hline CD24 & CD24 molecule & $1.1 \mathrm{E}-05$ & 11.5 \\
\hline PIGR & polymeric immunoglobulin receptor & $2.0 \mathrm{E}-05$ & 25.5 \\
\hline TMEM99 & transmembrane protein 99 & $4.6 \mathrm{E}-05$ & 2.1 \\
\hline PRLR & prolactin receptor & 0.0001 & 6.7 \\
\hline C8orf71 & chromosome 8 open reading frame 71 & 0.0001 & 0.1 \\
\hline PNRC2 & proline-rich nuclear receptor coactivator 2 & 0.0002 & 4.4 \\
\hline TRIM55 & tripartite motif-containing 55 & 0.0003 & 9.6 \\
\hline UGP2 & UDP-glucose pyrophosphorylase 2 & 0.0003 & 9.2 \\
\hline THBS1 & thrombospondin 1 & 0.0003 & 5.1 \\
\hline PF4V1 & platelet factor 4 variant 1 & 0.0003 & 2.8 \\
\hline VANGL1 & Vang-like 1 (van gogh, Drosophila) & 0.0003 & 2.5 \\
\hline LPHN1 & latrophilin 1 & 0.0003 & 0.3 \\
\hline ADD2 & adducin 2 (beta) & 0.0004 & 11.1 \\
\hline CDH23 & cadherin-like 23 & 0.0004 & 8.7 \\
\hline YKT6 & YKT6 v-SNARE homolog (S. cerevisiae) & 0.0004 & 3.7 \\
\hline TXNDC6 & thioredoxin domain containing 6 & 0.0004 & 0.1 \\
\hline TTC26 & tetratricopeptide repeat domain 26 & 0.0005 & 2.1 \\
\hline C3orf55 & chromosome 3 open reading frame 55 & 0.0005 & 0.4 \\
\hline GRIN3A & glutamate receptor, ionotropic, $N$-methyl-D-aspartate $3 A$ & 0.0006 & 6.2 \\
\hline C19orf33 & chromosome 19 open reading frame 33 & 0.0006 & 4.1 \\
\hline ZNF101 & zinc finger protein 101 & 0.0007 & 2.6 \\
\hline PTPRG & protein tyrosine phosphatase, receptor type, $G$ & 0.0007 & 2.1 \\
\hline ELF3 & E74-like factor 3 (ets domain, epithelial-specific) & 0.0008 & 3.6 \\
\hline SLC39A8 & solute carrier family 39 (zinc transporter), member 8 & 0.0008 & 2.3 \\
\hline FER1L4 & fer-1-like 4 (C. elegans) & 0.0008 & 2.2 \\
\hline $\mathrm{CP}$ & ceruloplasmin (ferroxidase) & 0.0009 & 4.8 \\
\hline SLC39A8 & solute carrier family 39 (zinc transporter), member 8 & 0.0009 & 3.4 \\
\hline STX3 & syntaxin 3 & 0.0009 & 2.8 \\
\hline FAM102B & family with sequence similarity 102 , member $B$ & 0.0009 & 0.4 \\
\hline
\end{tabular}

A similar but smaller analysis of acinar carcinoma cell-enriched and ductal carcinoma cell-enriched microdissected cell populations identified 30 characterized gene transcripts with significant differences in expression of at least two-fold between ductal and acinar adnocarcinomas (Table 2). However, this acinar versus ductal cell gene signature identified from microdissected cell populations still could not correctly classify acinar versus ductal tumors from whole sections, suggesting that intra-tumor cell heterogeneity is a significant confounding factor in defining a single molecular phenotype.

Of the 30 differentially expressed transcripts identified from the microdissected cell populations, expression of prolactin receptor transcript was elevated by approximately six-fold in ductal versus acinar carcinoma cells. To validate prolactin receptor protein expression in ductal versus acinar adenocarcinoma, immunohistochemistry was performed on 20 cases of adenocarcinoma of the prostate with both ductal and acinar components in the same case. For these 20 mixed acinar-ductal adenocarcinomas cases, the Gleason score of the ductal component was 8 (17 cases) or 9 (3 cases). The mean Gleason score of the acinar component was 6.8 (range 5 to 10). The pathologic T stage was pT2 in 7 cases and pT3 in 13 cases. Prolactin receptor immunostains showed diffuse and strong staining (immunoreactive score $\geqslant 4$ ) in the majority of ductal carcinoma regions $(15 / 20 ; 75 \%)$ (Figure 1a and b) as compared with acinar carcinoma regions $(4 / 20 ; 20 \%)$ (Figure $1 \mathrm{c}$ and d). The majority of acinar carcinoma regions showed no staining or weak and patchy staining at best (immunoreactive score $<4 ; 16 / 20$ ). Of the four cases of acinar carcinoma with immunoreactive score $\geqslant 4$, three had staining only in areas with Gleason grade 3 (Mean immunoreactive score $=6.7$, range: 6-8). The fourth case had Gleason score of 10 and showed moderate $(2+)$ staining in $50-79 \%$ of tumor cells (immunoreactive score $=6$ ). Mean immunoreactive score in regions of Gleason grade 4 acinar carcinoma was $1.1(n=13)$, compared with 4.17 for regions of Gleason grade $3(n=19)$.

\section{Discussion}

The data presented here show a high degree of molecular relatedness of ductal and acinar adenocarcinomas of the prostate, as assessed by gene expression profiling. Indeed, only 10-30 characterized gene transcripts across the entire assayed transcriptome were identified as significantly different in expression level when comparing ductal 

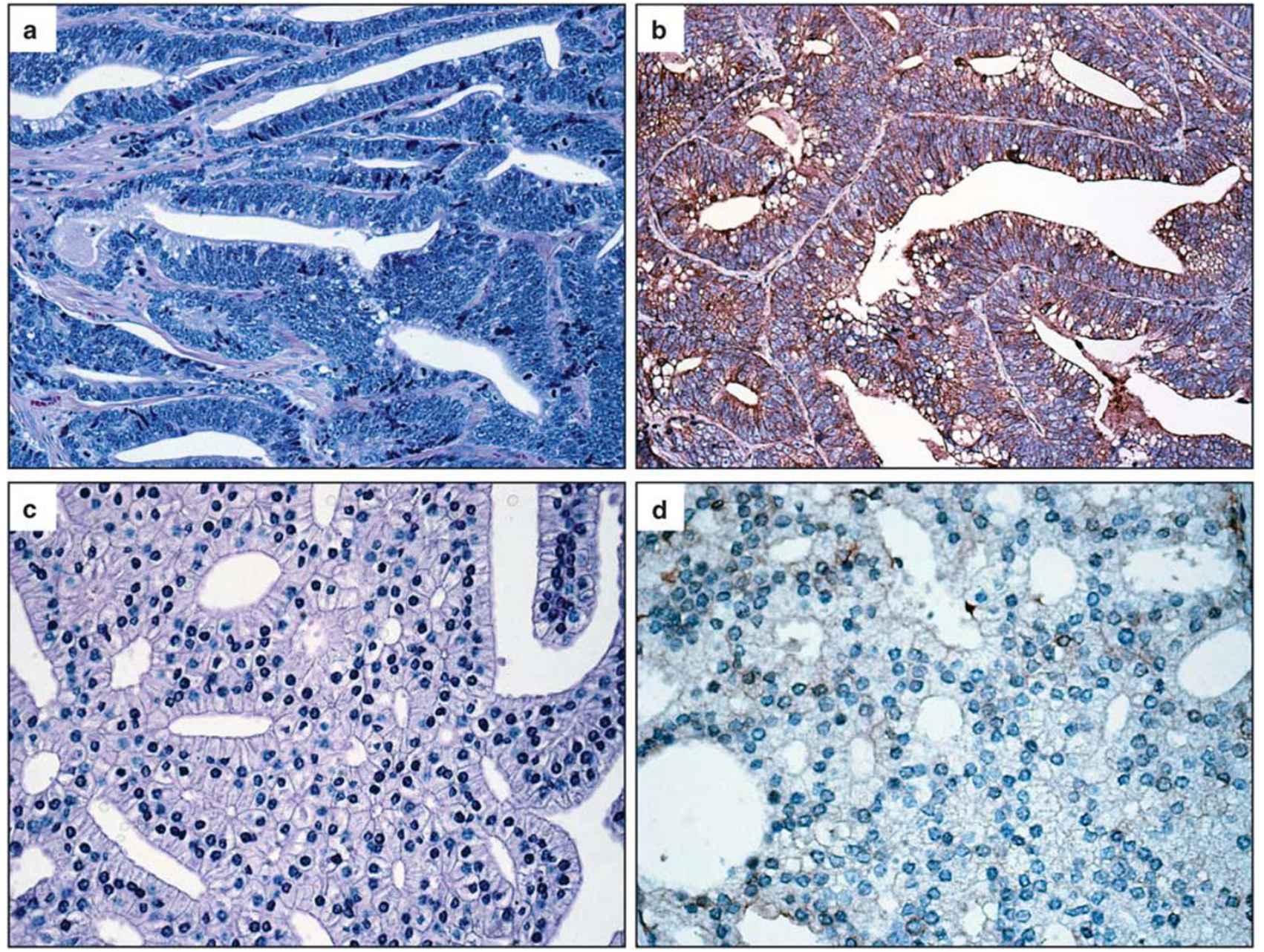

Figure 1 Prolactin receptor immunohistochemical staining of ductal (a, b) versus acinar (c, d) adenocarcinoma of the prostate. Image a (H\&E) and b (prolactin receptor immunostain), with b showing strong $(3+)$ cytoplasmic staining in $100 \%$ of ductal adenocarcinoma cells. Images from regions of acinar carcinoma with Gleason grade 4 in the same case show weak, focal staining by prolactin receptor immunostain (c: H\&E, d: prolactin receptor immunostain).

and acinar adenocarcinomas. Of interest, significant differences in gene expression were only apparent by microdissection of ductal and acinar carcinoma cell populations, reinforcing the importance of purification of cell types when gene expression analyses are performed. This result also highlights the heterogeneity of the whole prostate carcinoma tissue samples, with differing compositions of benign prostatic epithelial cells and different stromal cells (including fibroblasts, smooth muscle cells, myofibroblasts, nerves, endothelial cells, and inflammatory cells) that can influence the gene expression profile and confound profile comparisons of specific cell types.

Only a few studies have assessed the molecular relatedness of ductal and acinar adenocarcinomas of the prostate. ${ }^{29}$ The expression of only a few specific proteins has been evaluated by comparative immunohistochemistry. Similar to acinar adenocarcinomas, almost all ductal adenocarcinomas express prostate-specific antigen and prostate-specific acid phosphatase. ${ }^{15-17,20}$ Alpha-methylacyl CoA racemase (AMACR) expression has been detected in 58 to $100 \%$ of ductal adenocarcinomas. ${ }^{22-24}$ Although a lower percentage of cases of ductal adenocarcinoma seem to express AMACR compared with acinar adenocarcinoma, it has not been established whether there are comparative quantitative differences in AMACR protein levels. Another difference in immunophenotype is in Ki-67 labeling, in which the Ki-67 defined proliferation index for ductal adenocarcinoma is high, with a mean of $33 \%$, compared with usual acinar adenocarcinoma, at a mean of $8 \% .{ }^{25}$ In a small series, ductal adenocarcinomas displayed more frequent cytokeratin 20 expression. ${ }^{16}$ Immunohistochemical staining for other proteins, including estrogen receptor, androgen receptor, p53, bcl-2, and CEA, has been performed in a few ductal adenocarcinoma cases, ${ }^{7,14,18,19,30}$ but it is not established whether there is a difference in expression compared with acinar adenocarcinoma. 
Genes identified as exhibiting a greater than twofold change in ductal versus acinar adenocarcinoma are potential candidates for further study in attempts to explain the different histopathological features and disparate clinical behavior of ductal adenocarcinoma of the prostate. Of the 30 genes displaying a greater than two-fold difference in the microdissected samples, 10 were overexpressed greater than five-fold in ductal versus acinar adenocarcinoma and two were underexpressed greater than five-fold. Several of these genes have intriguing functions attributed to their protein products that could be important in ductal adenocarcinoma structure and biology. For example, CD24 (11.5-fold overexpressed in ductal adenocarcinoma) and cadherin-like 23 (8.8-fold overexpressed) have cell adhesion-related properties. CD24 is a potential oncogene reported to be overexpressed in a large variety of human malignancies. ${ }^{31}$ We selected the prolactin receptor for further characterization, as this polypeptide growth factor-receptor axis has been implicated in the development of the normal, ${ }^{32,33}$ hyperplastic, ${ }^{34}$ and neoplastic ${ }^{35}$ prostate. We found the prolactin receptor to be overexpressed in ductal versus acinar adenocarcinoma at both the transcript level and at the protein level, as validated by immunohistochemistry. Whether the prolactin receptor might affect ductal adenocarcinoma growth to a greater degree than acinar adenocarcinoma is not known, but it is of interest that prolactin promotes ductal morphogenesis ${ }^{34}$ and an increase in volume of ductal epithelium ${ }^{32}$ in rodent prostates. It should be noted that the prolactin receptor, although overexpressed in ductal compared with acinar adenocarcinoma, does not seem to be practical marker in immunohistochemistry for ductal adenocarcinoma, because of the overlap in immunopositivity in ductal and acinar adenocarcinomas.

In the future, the genes identified here as overexpressed in ductal versus acinar adenocarcinoma could serve as targets for development of markers of ductal adenocarcinoma. One approach would be to test additional antibodies, where available, in immunohistochemistry against the protein products of other overexpressed genes. A second approach could be the development of a quantitative RT-PCR assay, applicable using formalin-fixed, paraffinembedded tissues, for a panel of gene transcripts to distinguish ductal versus acinar adenocarcinoma.

On the basis of the results of this study, ductal and acinar adenocarcinomas of the prostate are highly related at the molecular level, as assessed by gene expression profiling, using both whole section and laser-capture microdissected cell populations. There is a small set of genes that exhibits a significant fold difference in expression level between ductal and acinar adenocarcinomas. Further research on these genes may provide insight into the basis for the specific histomorphological attributes and aggressive clinical behavior of ductal adenocarcinoma of the prostate.

\section{Disclosure/conflict of interest}

The authors declare no conflict of interest.

\section{References}

1 Grignon DJ. Unusual subtypes of prostate cancer. Mod Pathol 2004;17:316-327.

2 Humphrey PA. Variants of prostatic carcinoma (Chapter 17). In: Prostate Pathology ASCP Press: Chicago, 2003, pp 390-400.

3 Yang XJ, Cheng L, Helpap B, et al. Ductal adenocarcinoma In: Eble JN, Sauter G, Epstein JI, Sesterhenn IA, (eds) Tumours of the Urinary System and Male Genital Organs. IARC Press: Lyon, France, 2004, pp 199-201.

4 Young RH, Srigley JR, Amin MB, et al (eds). Variants of prostatic adenocarcinoma, other primary carcinomas of prostate, and secondary carcinomas (Chapter 5) and miscellaneous tumors of the prostate (Chapter 6). In: Tumors of the Prostate Gland, Seminal Vesicles, Male Urethra, and Penis, Third Series, Fascicle 28. Armed Forces Institute of Pathology: Washington DC, 2000;217-288.

5 Bock BJ, Bostwick DG. Does prostatic ductal adenocarcinoma exist? Am J Surg Pathol 1999;23:781-785.

6 Dube VE, Farrow GM, Greene LF. Prostatic adenocarcinoma of ductal origin. Cancer 1973;32:402-409.

7 Bostwick DG, Kindrachuk RW, Rouse RV. Prostatic adenocarcinoma with endometrioid features. Clinical, pathologic, and ultrastructural findings. Am J Surg Path 1985;9:595-609.

8 Brinker DA, Potter SR, Epstein JI. Ductal adenocarcinom of the prostate diagnosed on needle biopsy. Correlation with clinical and radical prostatectomy findings and progression. Am J Surg Pathol 1999; 23:1471-1479.

9 Samaratunga H, Delahunt B. Ductal adenocarcinoma of the prostate: current opinion and controversies. Anal Quant Cytol Histol 2008;30:237-246.

10 Lemberger RJ, Bishop MC, Bates CP, et al. Carcinoma of the prostate of ductal origin. Br J Urol 1984;56:706-709.

11 Christensen WN, Steinberg G, Walsh PC, et al. Prostatic duct adenocarcinoma. Findings at radical prostatectomy. Cancer 1991;67:2118-2124.

12 Epstein JI, Woodruff JM. Adenocarcinoma of the prostate with endometrioid features. A light microscopic and immunohistochemical study of ten cases. Cancer 1986;57:111-119.

13 Greene LF, Farrow GM, Ravits JM, et al. Prostatic adenocarcinoma of ductal origin. J Urol 1979;121:303-305.

14 Ro JY, Ayala AG, Wishnow KI, Ordoñez NG. Prostatic duct adenocarcinoma with endometrioid features: immunohistochemical and electron microscopic study. Semin Diagn Pathol 1988;5:301-311.

15 Gong Y, Caraway N, Stewart J, et al. Metastatic ductal adenocarcinoma of the prostate: cytologic features and clinical findings. Am J Clin Pathol 2006;126:302-309.

16 Leite KR, Mitteldorf CA, Srougi M, et al. Cdx2, cytokeratin 20, thyroid transcription factor 1 , and prostate-specific antigen expression in unusual subtypes of prostate cancer. Ann Diag Pathol 2008;12: 260-266.

17 Copeland JN, Amin MB, Humphrey PA, et al. The morphologic spectrum of metastatic prostatic adenocarcinoma to the lung: special emphasis on histologic 
features overlapping with other pulmonary neoplasms. Am J Clin Pathol 2002;117:552-557.

18 Oxley JD, Abbott CD, Gillatt DA, et al. Ductal adenocarcinoma of the prostate: a clinicopathologic and immunohistochemical study. Br J Urol 1998;81: 109-115.

19 Lee SS. Endometrioid adenocarcinoma of the prostate: a clinicopathologic and immunohistochemical study. J Surg Oncol 1994;55:235-238.

20 Millar EK, Sharma NK, Lessells AM. Ductal (endometrioid) adenocarcinoma of the prostate: a clinicopathologic study of 16 cases. Histopathology 1996;29:11-19.

21 Samaratunga H, Singh M. Distribution pattern of basal cells detected by cytokeratin 34betaE12 in primary prostatic duct adenocarcinoma. Am J Surg Pathol 1997;21:435-440.

22 Beach R, Gown AM, de Peralta-Venturina MN, et al. P504S immunohistochemical detection in 405 prostatic specimens including 376 18-gauge needle biopsies. Am J Surg Pathol 2002;26:1588-1596.

23 Herawi M, Epstein JI. Immunohistochemical antibody cocktail staining (p63/HMWCK/AMACR) of ductal adenocarcinoma and Gleason pattern 4 cribriform and noncribriform acinar adenocarcinomas of the prostate. Am J Surg Pathol 2007;31:889-894.

24 Wang H, Adley B, Keleman K, et al. Decreased expression of alpha-methylacyl coenzyme A racemase in prostatic ductal adenocarcinoma. Mod Pathol 2006;19(Suppl 3):89 (abstract).

25 Rioux-Leclercq N, Leray E, Patard JJ, et al. The utility of Ki-67 expression in the differential diagnosis of prostatic intraepithelial neoplasia and ductal adenocarcinoma. Hum Pathol 2005;36:531-535.

26 Sharma MK, Mansur DB, Reifenberger G, et al. Distinct genetic signatures among pilocytic astrocytomas relate to their brain region origin. Cancer Res 2007;67:890-900.

27 Benjamini Y, Hochberg Y. Controlling the false discovery rate: a practical and powerful approach to multiple testing. J Roy Sat Soc, Ser B 1995;57:289-300.

28 Tusher VG, Tibshirani R, Chu G. Significance analysis of microarrays applied to the ionizing radiation response. Proc Natl Acad Sci USA 2001;98:5116-5121.

29 Lotan TL, Toubaji A, Albadine R, et al. TMPRSS2-ERG gene fusions are infrequent in prostatic ductal adenocarcinoma. Mod Pathol 2009;22:359-365.

30 Tulunay O, Orhan D, Baltaci S, et al. Prostatic ductal adenocarcinoma showing Bcl-2 expression. Int J Urol 2004;11:805-808.

31 Kristiansen G, Sammar M, Altevogt P. Tumor biological aspects of CD24, a mucin-like adhesion molecule. J Mol Histol 2004;35:255-262.

32 Ingelmo I, Gomez V, Martin R, et al. Effect of prolactin and bromocriptine on the population of prostate neuroendocrine cells from intact and cyproterone acetate-treated rats: stereological and immunohistochemical study. Anat Rec 2007;290:855-861.

33 Nevalainen MT, Valve EM, Ingleton PM, et al. Prolactin and prolactin receptor are expressed and functioning in human prostate. J Clin Invest 1997;99: 618-627.

34 Kindblom J, Dillner K, Sahlin L, et al. Prostate hyperplasia in a transgenic mouse with prostatespecific expression of prolactin. Endocrinology 2003;144:2269-2278.

35 Dagvadorj A, Collins S, Jomain JB, et al. Autocrine prolactin promotes prostate cancer cell growth via Janus kinase-2-signal transducer and activator of transcription-5a/b signaling pathway. Endocrinology 2007;148:3089-3101. 